\title{
Sexual Signals in Electric Fishes
}

Electroreceptive bony fishes of Africa (the Mormyriformes) and South America (the Gymnotiformes) detect and communicate with conspecifics by their continuously discharging electric organs. Laboratory studies of members of each group are beginning to reveal the mechanisms of communicating with and finding mates, offering much scope for future studies of the behavioral ecology of electric fishes.

The ability to produce and detect weak electric currents, as a means of obtaining information about the environment, is one of the outstanding feats of evolution. It is found in two distantly related groups of tropical fishes: the elephant-nose fishes (Mormyriformes) of Africa, and the knife fishes (Gymnotiformes) of South America (Fig. 1). These fishes possess a highly sophisticated intelligence system, the capabilities of which continue to fascinate neurophysiologists, neuroanatomists and behavioral scientists; they have also become attractive subjects for students of evolution and ecology.

Weakly electric bony fishes, all of which seem to be predominantly nocturnal, test the impedance properties of their environment by setting up an electric dipole field for active electrolocation'. The electric discharges are produced by discrete electric organs (derived from modified muscle or, in one gymnotiform family, specialized motor nerve endings) and are detected by specialized lateral line receptors; the afferent electrosensory information is processed by a brain that is huge for a teleost (see reviews in Ref. 2).

In recent years, interest has focused on how electric fish detect mates and on the nature of the signals that are exchanged during courtship and spawning (reviewed in Ref. 3). Progress has been made both in the Mormyridae*, whose electric discharge takes the form of

*Mormyrids also communicate acoustically. A territorial male defending a nest produces an acoustic song ${ }^{4}$ that is strong in the nights preceding courtship; the song also occurs for about one hour immediately after dark of a spawning night, it wanes rapidly during courtship (the next two hours), and it is absent during the long subsequent spawning phase (about four more hours) ) $^{5}$ Mormyrids have keen hearing ${ }^{6.7}$; females may be attracted by these calls from a distance.

Bernd Kramer is at the Zoological Institute of the University, D-8400 Regensburg, FRG.

\section{Bernd Kramer}

a pulse, and in a few gymnotiform fishes, which use either a wave or a pulse type of discharge.

Intraspecific variability of pulse-type discharges in a mormyrid and a gymnotiform

The electric organ discharge (EOD) of the mormyrid Pollimyrus isidori is a brief pulse, with two positive $(P)$ phases separated by a negative (N) phase (Fig. 2a). This pulse is repeated at a low and variable rate of about 10-100 pulses per second (p.p.s.) when the fish is excited (a 'buzzer'). The waveform of this pulse-EOD shows great intraspecific variability $^{8}$, suggesting that the EOD waveform might be a sexual cue'. A similar hypothesis had been advanced by Hopkins and Bass ${ }^{10}$ for an as yet unidentified mormyrid, Brienomyrus sp.; unfortunately, there is no full report (see review in Ref. 11), and the hypothesis of sexual recognition by pulse waveform needs further experimental support ${ }^{12,13}$.

There is a statistically significant tendency for female $P$. isidori to show an EOD waveform different from that of males (Fig. 2a); however, there is considerable overlap between the two distributions, and no clear decision concerning the sex of an individual on the basis of its EOD waveform is possible for most fishes $^{13}$. Furthermore, because two females with 'wrong' EOD waveform or duration have been observed spawning repeatedly in the laboratory, it appears that the EOD waveform information is not critical for pair formation or spawning in $P$. isidori $^{5}$.

An interesting comparison can be made with the gymnotiform Hypopomus occidentalis from Panama, the discharge of which roughly resembles a single-cycle sinusoidal pulse. The discharges of males tend to be stronger and to last longer than those of females. Despite considerable overlap, the difference between the distributions for the two sexes is statistically significant ${ }^{14}$. Females give more discharge-rate responses ('decrement bursts'15; see below) to being stimulated with single-cycle sinusoids of male, rather than female, discharge duration $^{16}$. Of the wide range of spectral components of Hypopomus' pulse discharge, it is only the frequency of peak amplitude that the fish seem to need for mate recognition ${ }^{16}$. These fish differ from mormyrids in displaying a usually constant, and rather high, pulse rate ${ }^{15}$.

Effects of water conductivity on mormyrid discharge waveform

The EOD waveform of $P$. isidori land, because of the general biophysical principles ${ }^{17,18}$ involved, probably all mormyrids) depends on the conductivity of surrounding water $^{13}$ (Fig. 2b). For P. isidori, conductivity in its natural West African habitat is about $20-110 \mu \mathrm{S} \mathrm{cm}^{-1}$. Water conductivity is generally still lower in the equatorial Zaïre region where most mormyrid species live occasionally extending down to $5 \mu \mathrm{S}$ $\mathrm{cm}^{-1}$ (Ref. 3) (i.e. a conductivity almost equivalent to that of the deionized water often used in laboratories).

The dependence of discharge waveform on conductivity is weak in some individuals of $P$. isidori and strong in others; therefore, there is no simple correlation of EOD waveform change with conductivity that might enable fish to 'read' EOD waveform under some standard conductivity if the actual water conductivity were known to the fish.

Mormyrid electric organs are derived from skeletal muscle, a tissue known to respond strongly to
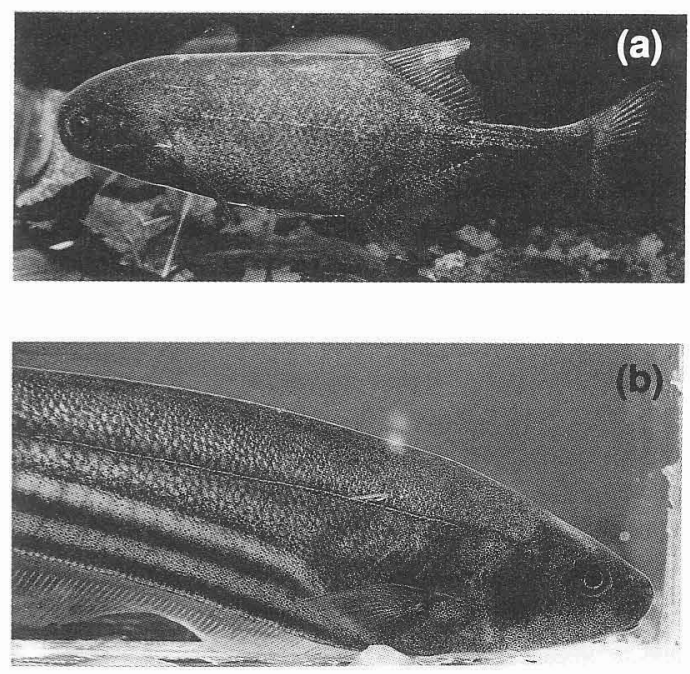

Fig. 1. (a) The African weakly electric mormyrid Pollimyrus isidori (up to $9 \mathrm{~cm}$ in length). Photograph courtesy of G. von der Emde. (b) A large male of the South American weakly electric knife fish Eigenmannia sp. (males up to $40 \mathrm{~cm}$, females up to $20 \mathrm{~cm}$ ). 

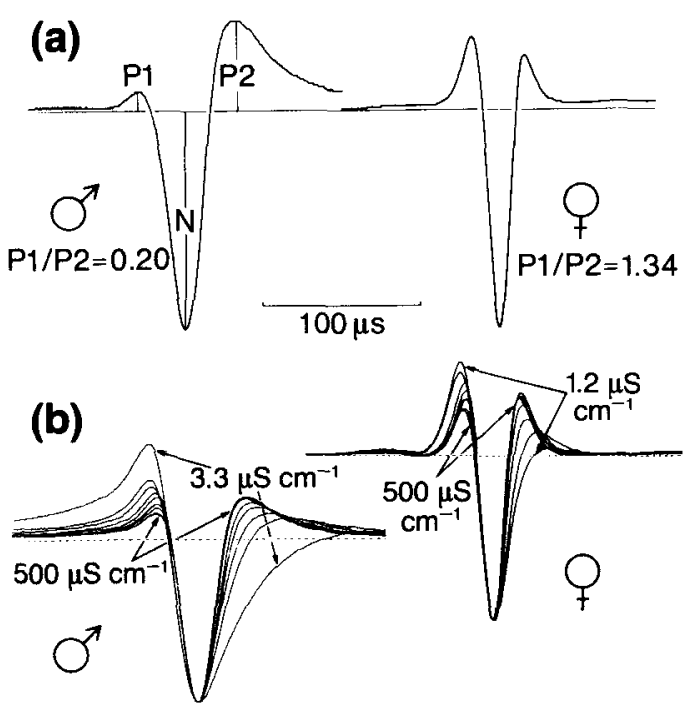

Fig. 2. (a) Electric organ discharges, of the pulse type, of a male and a female Pollimyrus isidori, digitized at $2 \mathrm{MHz}$. The two head-positive (P) phases are separated by the negative (N) phase. In males, the amplitude of the PI phase tends to be lower than that of the P2 phase ii.e. $\mathrm{Pl} / \mathrm{P} 2<1$ ); in females, the reverse tends to be true (P1/P2 $>1$ ). (b) A male and a female organ discharge of Pollimyrus isidori, recorded through a range of water conductivities: 1.2 or $3.3,5,10,20,50,100,200,500 \mu \mathrm{S}$ $\mathrm{cm}^{-1}$. |Conductivity, measured in siemens $(S)$ per $\mathrm{cm}$, is the equivalent of the inverse resistivity $(\Omega \mathrm{cm})^{-1}$. I Note that with increasing water conductivity the first $P$ phase decreases in amplitude while the second $P$ phase increases, and the duration of the $\mathrm{N}$ wave becomes shorter. From Ref. 13.

androgen hormones and their anabolic effect. This effect is probably the reason for the statistical trend of $P$. isidori males to have stronger EODs and a lower P-ratio ${ }^{13}$ (Fig. 2a). Mature males of another mormyrid, Gnathonemus petersii, tend to have a longer EOD than females ${ }^{19}$, although this trend cannot be seen in gonadally mature subadults ${ }^{20}$. The effect of androgen hormones on mormyrid electric organs is reviewed by Bass ${ }^{21}$.

\section{Mormyrid courtship and spawning signals encoded as inter-pulse interval patterns}

Recently, the electric discharge patterns that accompany the nocturnal courtship and spawning of $P$. isidori have been recorded in the laboratory. Specific EOD interval patterns were firmly correlated with diverse motor acts, suggesting that these EOD patterns are important for mutual acceptance of mates to proceed on to the most critical stages of reproductive behavior ${ }^{5}$ For example, during courtship and spawning bouts the male and female lock into a regular interval pattern of low and constant rate punctuated by a few pauses during the moments of vent-to-vent coupling. Such a pattern is exceedingly unusual in an interacting pair of $P$. isidori, except during courting or spawning. Both partners take on completely different roles, associated with specific interval patterns: the male is territorial and builds a nest, guarding the eggs and young fry; the female is allowed access to the nest area only during the brief courtship and spawning bouts (1525 s each; repeated for hours on a spawning night).

Another way in which $P$. isidori
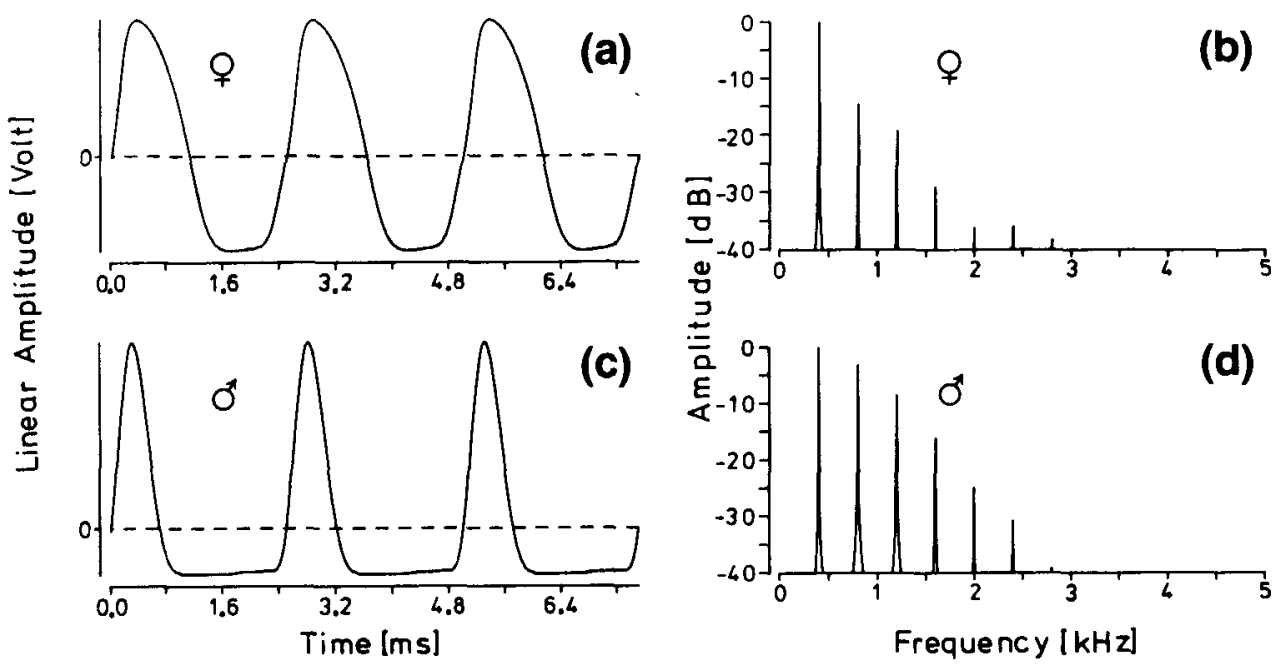

Fig. 3. (a. $\mathrm{c}$ ) Electric organ discharges, of the wave type, of a female and a male Eigenmannia sp. (species range, $250-600 \mathrm{~Hz}$. Note the marked difference in the time pattern of zero-crossing intervals: in the female, head-positive and negative half-waves are of almost equal duration, while in the male the headnegative half-wave is much longer than the positive.

(b, d) Fourier amplitude spectra; the fundamental frequency, of $400 \mathrm{~Hz}$ in these examples, is expressed as $0 \mathrm{~dB}$ attenuation. $(\mathrm{A}$ decibel is a logarithmic measure of intensity relative to some arbitrary reference intensity of $0 \mathrm{~dB}$; compared to the reference intensity, an attenuation of $-20 \mathrm{~dB}$ is an intensity drop to one tenth, of $-40 \mathrm{~dB}$ to one hundredth, and so forth.) Note the lower intensity of overtones lof frequency $400 n$, where $n$ is $2,3,4 \ldots$ in the female discharge as compared to the male discharge. From Ref. 12. males and females differ is in a rapid reflex-like, purely electrical response to a conspecific - the preferred latency response (PLR) of males and the preferred latency avoidance (PLA) of females ${ }^{8,22}$. Males tend to respond to another fish's EOD by discharging at a latency of $12 \mathrm{~ms}$, while females avoid this latency range by delaying their response EOD for at least $20 \mathrm{~ms}$. The behavioral significance of this response, also found in other mormyrids (mostly in the form of PLR), is unclear.

In summary, while the waveform of the EOD pulse of $P$. isidori does not seem to carry critical information despite its great intraspecific variability, the inter-EOD interval patterns clearly do. This is shown by the stereotyped correlation of a wealth of specific patterns with specific reproductive ${ }^{5}$ and nonreproductive $^{5,22}$ overt behaviors, and the male's predictable switching of pattern whenever the female enters or leaves his nesting territory. A similar conclusion was derived from a playback study in the context of agonistic behavior in the mormyrid G. petersii ${ }^{23}$.

The pulse-discharging gymnotiform $H$. occidentalis also displays discharge-rate modulations during courtship and spawning. The 'decrement burst' is a very brief acceleration of the fish's otherwise steady discharge rate ${ }^{15}$; a more detailed description of the relationship with overt behavior is, however, lacking.

\section{Intraspecific variability of a wave-type} discharge in a gymnotiform

Intraspecific variability in discharge waveform clearly plays a role in the South American gymnotiform Eigenmannia (Sternopygidae), whose EOD is a wave of usually constant frequency (a 'hummer'). Adult females and juveniles have an EOD waveform that resembles a sinusoid much more closely than does that of adult males; the latter produce narrow head-positive pulses superimposed on a negative baseline ${ }^{12}$ (Fig. 3). Accordingly, the intensity of higher harmonics, or overtones, is high in adult males and lower in other individuals. Gottschalk described a similar waveform variation in the related Sternopygus (reviewed in Ref. 12).

Food-rewarded Eigenmannia 
have been found to discriminate between electronically synthesized male and female discharges ${ }^{24}$. Moreover, in a simultaneous choice experiment without reward or punishment, untrained juvenile and adult fish of both sexes preferred to move to and stay close to an electric dipole emitting female EODs of constant frequency land amplitude), rather than a dipole emitting male EODs ${ }^{25}$. This experiment confirmed that fish can discriminate male from female EOD waveforms, with neither intensity nor frequency being factors; the experiment also showed that the difference matters to the fish even in a nearly bare aquarium, outside a reproductive context.

Unlike the pulse discharge of $P$. isidori, Eigenmannia's discharge waveform is affected very little by conductivity change (within the natural range of water conductivities, $10-100 \mu \mathrm{S} \mathrm{cm}^{-1}$ ); the reason for the difference is the entirely different physiology of their electric organs ${ }^{17.18}$ (reviewed in Ref. 25). Also, temperature change (within the range $20-33^{\circ} \mathrm{C}$ ) has very little effect on Eigenmannia's discharge waveform, apart from on its frequency $^{25}$.

\section{Signal modulations of 'hummers' during reproductive behavior}

In Eigenmannia, frequency modulations of its usually constantfrequency wave discharge, and brief interruptions, have been observed in interacting mature fish and during spawning in the laboratory ${ }^{26}$. On becoming territorial in a large tank, a large male begins to 'chirp' routinely at night, at a rate between one per minute and five per second. A 'chirp' is a brief discharge interruption (Fig. 4) that is displayed both in an agonistic context towards other males ${ }^{27}$, and during courtship ${ }^{26}$. In response to mild attacks of the much larger male, a female will repeatedly and smoothly raise her discharge frequency by a few to several hertz over a period of tens of seconds ('long rise'). Long rises are considered a submissive signal in an agonistic context ${ }^{27}$, but seem to play an additional role in courtship in stimulating the male to increase his rate of chirping. Also observed during the night of spawning are 'short rises', displayed by both sexes.

(a)

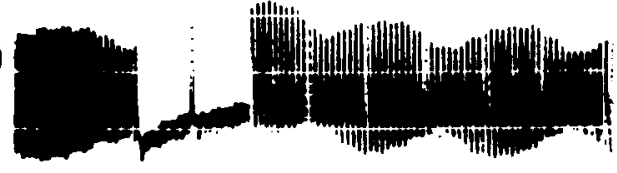

A female will only spawn when a male has chirped at her site for at least one hour. Playback of tape recordings of a courting male through a dipole also evoked spawning by a gravid female in the vicinity of the electrodes ${ }^{26}$.

In Sternopygus macrurus, males and females tend to discharge at a frequency difference of an octave ${ }^{27}$. Different frequency ranges have also been observed in the two sexes of Eigenmannia 26.12 and of Apteronotus leptorhynchus, a gymnotiform fish that also chirps during spawning ${ }^{26}$. It is, however, not known whether a certain minimum frequency difference is necessary for spawning to occur in a certain pair of fish; in Eigenmannia spawning in the laboratory this does not seem to be the case ${ }^{28}$.

\section{Sexual dimorphism of Eigenmannia's jamming avoidance response}

A special kind of frequency modulation is the so-called jamming avoidance response (JAR ${ }^{29.30}$ ). To avoid 'jamming' of its signal by a conspecific, a 'model' Eigenmannia ought to lower its own frequency in response to stimuli of slightly higher frequency, and increase its own frequency in response to stimuli slightly lower, so that the difference between the two frequencies (the beat frequencyl increases. However, the reality is more complex (Fig. 5) ${ }^{31}$

The JAR is usually thought to help maintain a fish's performance of active electrolocation in the presence of disturbing noise lof similar frequencyl from a conspecific's discharge (see Ref. 32), but at the same time some authors suggest a social or communicative role ${ }^{33}$.

The recently discovered sexual dimorphism of JAR strengthens that view $^{31}$ (Fig. 5). Adult males do not respond to jamming signals by displaying a JAR, but instead produce short interruptions ('chirps') or 'short rises'. Adult females, discharging usually at the highfrequency end of the species' range only lower their frequency, and do not respond to stimulus frequencies that should evoke a frequency increase. Females may, however, increase their frequency with a time course resembling a JAR in an agonistic context (submissive signal ${ }^{27}$ ), or during courtship in the presence of males. Juveniles, finally, respond
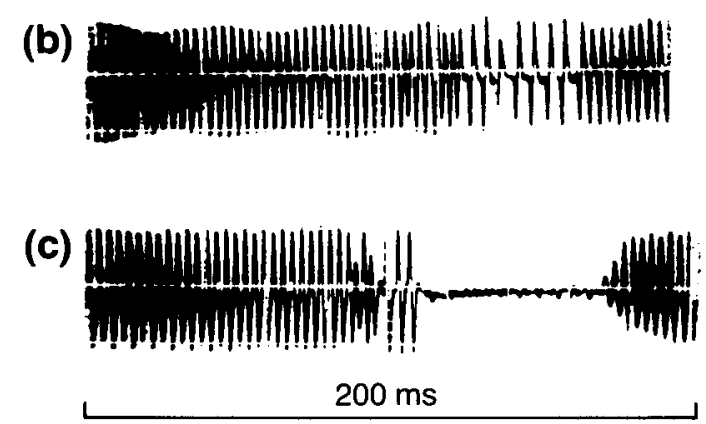

Fig. 4. Oscillograms of discharge interruptions, or 'chirps', of a male Eigenmannia virescens. (a) A sudden, almost complete interruption, as observed during agonistic behavior. (b) An incomplete interruption, as observed during courting. (c) A long and complete interruption following an initially incomplete interruption. Adapted from Ref. 26, with permission.

in both directions, but deviate from the 'model' Eigenmannia either by shifting their discharge frequency in the 'wrong' direction in response to a stimulus frequency slightly higher than their own, or by a markedly asymmetric response strength to equal difference frequencies of opposite sign. These two groups probably represent the two sexes ${ }^{31}$ (see Fig. 51.

Although the explanation of the IAR as adaptive for active electrolocation is plausible and attractive, the evidence is not particularly strong (see Ref. 31). An alternative perhaps not mutually exclusive explanation ${ }^{31}$ would argue that the JAR is a frequency modulation that assures a private frequency 'window' for all members of a school lexcept large males, which do not show the response), so that a fish can better detect and analyse the sex- and age-correlated EOD waveforms (Fig. 3) and frequency modulations (Figs 4,5 ) of other fish, and the spatial structure of the group. Under the new hypothesis, a fish would not be trying to minimize, but rather to maximize, the effect of another fish's discharge on its own, by shifting its own frequency by a few hertz to an optimal beat frequency.

\section{Ecology of reproductive cycles}

Kirschbaum (see Ref. 34) found that by imitating some ecological changes that precede the reproduction of many Amazonian fishes, some weakly electric fishes (such as Eigenmannia virescens and also the 

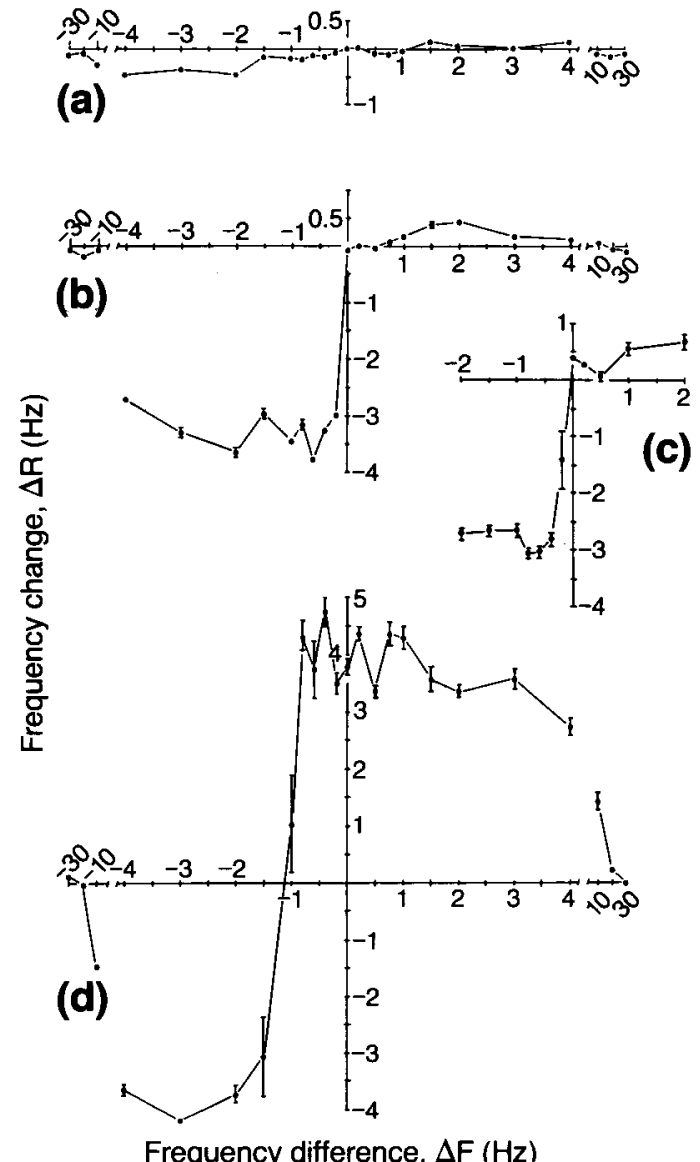

Fig. 5. The frequency change $(\Delta R)$ displayed by four Eigenmannia individuals in response to stimuli of similar frequency. $\Delta \mathrm{R}$ is measured as the fish's response frequency minus its resting frequency; it is plotted here as a function of the frequency difference $(\Delta F)$, i.e. the fish's resting frequency minus the constant stimulus frequency. Each point is a mean of 12 measurements, $\pm 1 \mathrm{sE}$.

Large males (a) barely respond at all, even at increased stimulus intensities $1+10$ and $+20 \mathrm{~dB}$; not shown). Adult females (b) readily lower their frequency to negative $\Delta \mathrm{Fs}$, but show no or only weak responses to positive $\Delta F$ s. luveniles or subadults respond in both directions; however, those that are presumably females (c) only give weak frequency increases to positive $\Delta F s$, while the other type of juvenile (d), which is probably the males, gives strong responses to both positive and negative $\Delta F s$, including $\Delta F=0 \mathrm{~Hz}$. This type of juvenile even increases its frequency when the stimulus frequency is slightly higher than its own (negative $\Delta \mathrm{Fs}$ of small absolute value up to about $\mathrm{I} \mathrm{Hz}$ ). For further experimental details, see Ref. 31 .

African $P$. isidori) can be induced to spawn in the laboratory. Chief among these factors was a slow lowering of water conductivity, as naturally occurs (following an initial increase) in the Amazon (Manaus) at the start of the rainy season. At this time, large nutrient-rich inundation areas become available as spawning grounds for many fish species ${ }^{35}$. Because of their temporal cor- relation, conductivity changes together with a rise in water level would work well as stimuli for reproduction in some, but not all, fish communities. In some cases there is no clear pattern of rainy and dry seasons ${ }^{35}$; in others, there is such a pattern but no appreciable change of water conductivity can be recorded throughout the year ${ }^{36,15}$.

Schwassmann (reviewed in Refs 27,15 ) has suggested that the dryseason spawners of the subtropical zones (more than $9-10^{\circ} \mathrm{N}$ or S) may respond to diurnal cues for the control of their reproductive cycles, while the wet-season spawners of the more equatorial regions re spond to physical or chemical cues of the water.

Many tropical fish species perhaps respond opportunistically to the availability of sufficient highquality food, and suitable spawning grounds, by growth of their ova. A more parsimonious explanation such as this does not conflict with most of the available data on the environmental control of reproduction in weakly electric fishes (and perhaps other fishes as well). The occasional spontaneous spawnings in aquaria at high conductivities (that were probably rather rising than falling), like the first such observation by Birkholz (see Ref. 34) in $P$. isidori, may also lend some weight to this possibility. In any case, the laboratory studies described here are now generating many interesting questions about the behavioral ecology of weakly electric fishes.

\section{References}

1 Lissmann, H.W. (1958) J. Exp. Biol. 35 156-191

2 Bullock, T.H. and Heiligenberg, W., eds (1986) Electroreception, Wiley

3 Kramer, B. Electrocommunication in Teleost Fishes: Behavior and Experiments. Springer-Verlag (in press)

4 Crawford, I.D., Hagedorn, M. and Hopkins, C.D. (1986) /. Comp. Physiol. 159, 297-310 5 Bratton, B.O. and Kramer, B. (1989) Behav. Ecol. Sociobiol. 24, 349-368

6 Kramer, B., Tautz, I. and MarkI, H. (1981)

J. Comp. Physiol. 143, 435-44I

7 McCormick, C.A. and Popper, A.N. (1984)
1. Comp. Physiol. 155, 753-761

8 Lücker, H. and Kramer, B. (1981) Behav. Ecol. Sociobiol. 9, 103-109

9 Westby, G.W.M. and Kirschbaum, F. (1982)

J. Comp. Physiol. 145, 399-403

10 Hopkins, C.D. and Bass, A.H. (1981)

Science 21 2, 85-87

11 Hopkins, C.D. (1988) in Sensory Biology of Aquatic Animals (Atema, J., Fay, R.R.

Popper, A.N. and Tavolga, W.N., eds), pp. 233-268, Springer-Verlag

12 Kramer, B. (1985) /. Exp. Biol. 119, 41-69

13 Bratton, B.O. and Kramer, B. (1988) Exp. Biol. 47, 227-238

14 Hagedorn, M. and Carr, C.E. (1985)

J. Comp. Physiol. 156, 511-523

15 Hagedorn, M. (1988) Copeia 1988

324-335

16 Shumway, C.A. and Zelick, R.D. (1988)

I. Comp Physiol 163, 465-478

17 Bennett, M.V.L. (1971) in Fish Physiology (Vol. V) (Hoar, W.S. and Randall, D.J., eds) pp. 347-491, Academic Press

18 Bell, C.C., Bradbury, J. and Russell, C.J. (1976) J. Comp. Physiol. 1 10, 65-88

19 Landsman, R.E. and Moller, P. (1988) Experientia 44, 900-903

20 Kramer, B. and Westby, G.W.M. (1985) Experientia 4I, 1530-1531

21 Bass, A.H. (1986) in Electroreception (Bullock, T.H. and Heiligenberg, W., eds) pp. 13-70, Wiley

22 Kramer, B. (1978) Behav. Ecol. Sociobiol $4,61-74$

23 Kramer, B. (1979) Behav. Ecol. Sociobiol. $6,67-79$

24 Kramer, B. and Zupanc, G.K.H. (1986)

Naturwissenschaften 73,679-680

25 Kramer, B. and Otto, B. (1988) Behav. Ecol. Sociobiol. 23, 55-60

26 Hagedorn, M. and Heiligenberg, W.

(1985) Anim. Behav. 33, 254-265

27 Hagedorn, M. (1986) in Electroreception (Bullock, T.H. and Heiligenberg, W., eds). pp. 497-525, Wiley

28 Westby. G.W.M. and Kirschbaum, F. 11981 ) in Sensory Physiology of Aquatic Lower Vertebrates (Adv. Physiol. Sci., Vol 31 ) (Szabo, T. and Czeh, G., eds), pp. I79194, Pergamon Press/Akademiai Kiado 29 Watanabe, A. and Takeda, K. (1963) I. Exp. Biol. 40, 57-66

30 Bullock, T.H., Hamstra, R.H. and Scheich, H. ( 1972) J. Comp. Physiol. 77, 1-48

31 Kramer, B. (1987) /. Exp. Biol. 130, 39-62

32 Heiligenberg, W. (1986) in

Electroreception (Bullock, T.H. and

Heiligenberg, W., edsI, pp. 613-649, Wiley 33 Scheich,H. ([977) ). Comp. Physiol. 113, $181-255$

34 Kirschbaum, F. (1984) Environ. Biol. Fishes 10, 3-14

35 Sioli, H., ed. (1984) The Amazon: Limnology and Landscape Ecology of a Mighty Tropical River and its Basin, Junk Publishers

36 Westby, G.W.M. (1988) Behav. Ecol. Sociobiol. 22, 341-354 